\title{
Henri VI : le mythe contre l'histoire
}

\section{Pierre Sahel}

\section{(2) OpenEdition \\ Journals}

Édition électronique

URL : http://journals.openedition.org/shakespeare/493

DOI : 10.4000/shakespeare.493

ISSN : 2271-6424

Éditeur

Société Française Shakespeare

Édition imprimée

Date de publication : 1 novembre 1984

Pagination : 51-66

Référence électronique

Pierre Sahel, « Henri VI: le mythe contre l'histoire », Actes des congrès de la Société française

Shakespeare [En ligne], 5 | 1984, mis en ligne le 01 janvier 2007, consulté le 21 avril 2019. URL : http:// journals.openedition.org/shakespeare/493; DOI : 10.4000/shakespeare.493 
SOCIETE FRANÇAISE SHAKESPEARE

Actes du Congrès 1983

\section{MYTHE ET HISTOIRE}

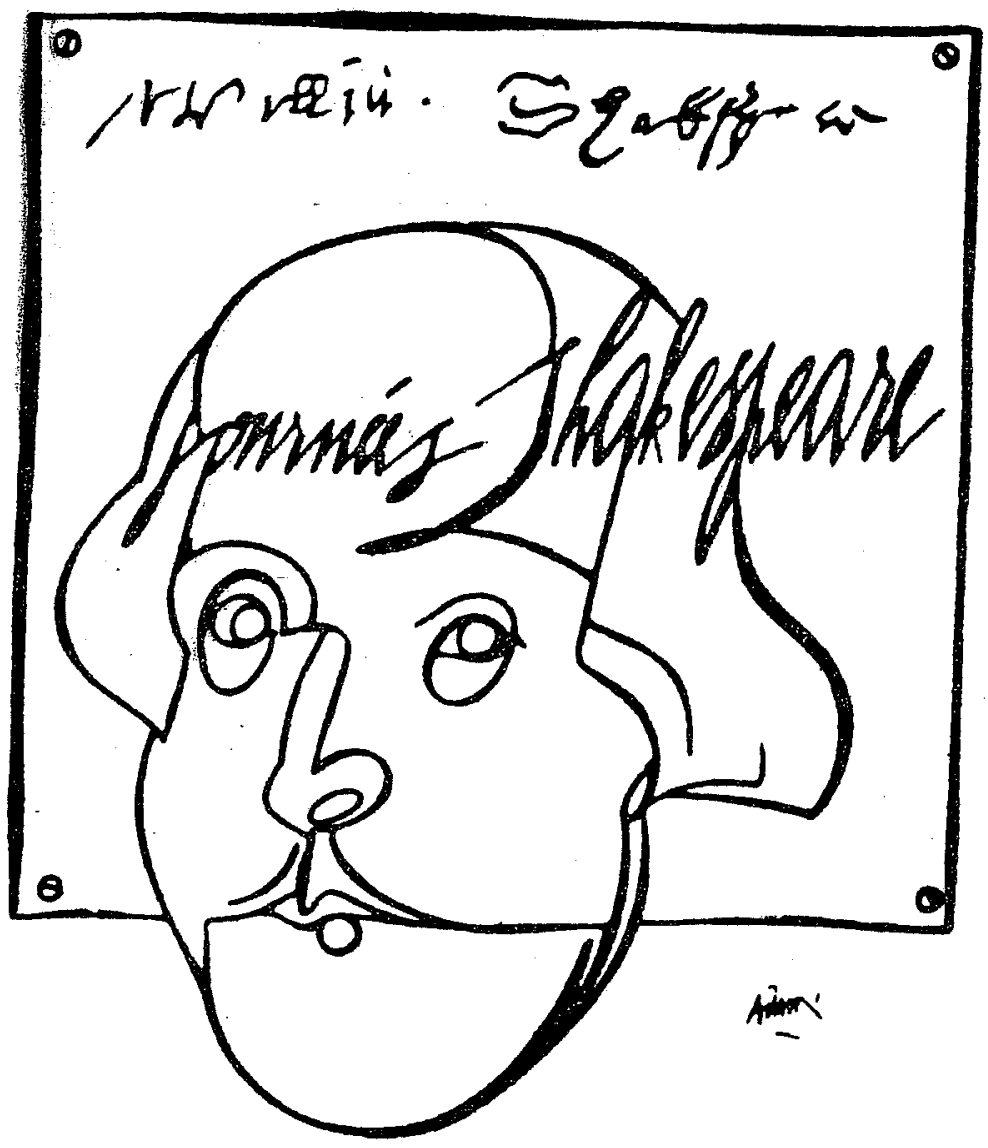

$$
\begin{array}{r}
\text { DiReCteur DE LA' PUblicatic } \\
\text { M.T. Jones-Davie }
\end{array}
$$

Publié avec le concours du Centre National de la Recherche Scientifiq

$$
\text { JEAN TOUZOT Libraire - Editeur }
$$

38, rue Saint-Sulpice 75278 PARIS CEDEX 06199 


\section{HENRI VI: LE MYTHE CONTRE L'HISTOIRE}

En associant dans mon titre les termes «mythe» et «histoire» qui constituent cette année le thème de réflexion de notre Société, je suis conscient du poids des mots comme de l'apparente nécrssité de répondre à l'attente de l'élucidation lexicale et de l'énoncé de critères méthodologiques. Pourtant les définitions tant de «mythe» que $d^{\prime}$ '《histoire» sont multiples, plus fines et plus précises les unes que les autres; et c'est l'impérialisme des méthodes et des approches qui m'incite à refuser au principe l'agencement dans des cadres rigides de ce que j'entends par «mythe» et par «histoire» dans les trois «Parties» de Henri $V I$. Pour moi (mais certainement pour d'autres aussi), l'histoire, dans Henri VI (mais certainement ailleurs aussi), c'est un ensemble d'événements relatifs à l'évolution passée d'un groupe social et qui sont jugés dignes d'être conservés dans la mémoire en étant, par exemple, théâtralisés. Il me semble aller de soi qu'aucun dramaturge ne se livre à une enquête scientifique pour écrire l'histoire. Shakespeare veut seulement faire «passer» le contenu de ses pièces : il soulève des passions, invite à la réflexion, suscite des émotions. Même s'il introduit dans ses oeuvres une foule de références sociales, il n'ambitionne pas de prendre en compte la totalité de la condition humaine d'une période disparue - ce qui est un des idéaux de l'historien. L'exploration et la signification d'expériences individuelles, dès Henri VI, importent infiniment plus que tous les éléments de pensée, politiques ou autres, dont ses oeuvres sont truffées. Par «mythe» j'entends d'abord simplement ce que l'on désigne habituellement par «mythe» ou par «lêgende» lorsque l'on parle du mythe d'Icare ou du mythe de Prométhée : un récit fabuleux comportant une symbolisation d'un ou de plusieurs aspects de la condition humaine. Mon titre lui-même ne veut rien avoir de paradoxal car le mythe (en général : prenons l'exemple de la guerre de Troie dans Homère), à l'inverse de l'histoire, n'est pas relié par le 
temps ou par d'autres contextes à ce qui devait «arriver ensuite» : il se contente de fonder sa narration sur un petit nombre de faits ayant ou acquérant une signification universelle ${ }^{1}$.

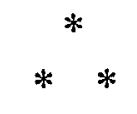

Dans les Henri VI, Shakespeare n'a pas voulu montrer que le détenteur nominal de la plus grande puissance, le roi, était le moteur le plus efficace de 1'histoire. Il s'est parfois soucié de dramatiser l'inverse : l'addition de la faiblesse réelle à la puissance théorique. Nul ne songerait à voir en Henri VI un des artisans de l'histoire des drames qui portent son nom. A travers le voile de la piété du personnage, transparaît certes un monde dans lequel Dieu provoque les moindres événements. Il y a beaucoup de candeur et de naïveté dans cette attitude - beaucoup de danger aussi, car son préjugé «providentialiste» conduit Henri VI à se croire le déchiffreur des voies de Dieu. Chaque fois que les événements lui échappent, que, du fait de ses partisans ou de ses ennemis, ils tournent à son avantage ou à son désavantage, ils sont assignés à la bonté ou au courroux de Dieu. Le portrait dramatique de Henri VI n'est guère tragique parce que le protagoniste conserve sa piété amorphe et sa passivité quand d'autres, autour de lui, utilisent la ruse, conpirent et biaisent. C'est que l'histoire, au long des Henri VI, est une histoire dure, faite d'actes cruels, de complots, de calculs, de passes veules, d'échecs, d'élans brisés, point du tout une marche triomphale qu'il s'agit de célébrer en la dramatisant. C'est, dans 1 Henri VI, la perte lamentable des chères conquêtes françaises causée par la désunion et l'égoïsme des nobles anglais, causée aussi par le manque d'hommes et d'argent. C'est, dans 2 Henri VI, le déclenchement de complots et de contrecomplots, la révolte armée de Richard d'York, l'assassinat de Gloucester , le chantage . . ., le tout sur un fond de chicanes nobiliaires et de jérémiades royales. L'histoire, dans 3 Henri VI, c'est la guerre civile, les moqueries infligées 
aux mourants et aux morts, l'échec de l'éphémère «compromis historique» où l'on a proclamé : «Now York and Lancaster are reconcil'd (I. i. 210, ed. Andrew S. Cairncross) ; c'est encore la lamentable négociation matrimoniale menée à la cour de France, l'érotisme dangereusement insouciant du roi Edouard, le couronnement et le découronnement des chefs des deux Maisons rivales. . Histoire désordonnée, faite de contes pleins de bruit et de fureur, sans dessein didactique ou téléologique ; histoire ironique ; histoire réaliste aussi, en ce sens qu'elle s'écrit épisodiquement et que les menées des uns et les ambitions des autres s'opposent et s'annulent comme les mines et les contre-mines dont parle Hamlet. Il n'y a rien d'étonnant à ce que les images des trois pièces illustrent ou soulignent la désintégration des valeurs et des personnages eux-mémes $^{2}$. Dans 1 Henri VI, le monde humain se peuple de bêtes de proie à la curée ; l'homme y est le frêle animal sans cesse menacé de tomber dans un piège. Dans 2 Henri $V I$, l'homme n'est plus l'animal menacé mais la bête menaçante qui traque ou pique; York y devient une araignée ou un serpent, l'Angleterre, un nid de scorpions. 3 Henri VI, à son tour, est envahi de faucons, d'insectes, de renards, de loups, de lions ... qui assaillent agneaux et oiseaux innocents. Les personnages se désagrègent, comme York, le presque roi, agenouillé et sanglotant aux pieds de Marguerite, la presque femme, qui le coiffe d'une couronne de papier et de force à essuyer ses larmes avec un mouchoir teinté du sang du plus jeune de ses enfants.

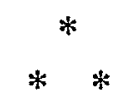

Pour un temps, malgré tout, la première «Partie» de Henri VI, du moins, peut laisser croire à la mythisation du héros dans l'histoire ${ }^{3}$. Pour un temps, les héros sont les soldats anglais, surhommes associés à Roland, à Olivier, à Samson, à Goliath (I.ii. 30-33, ed. Andrew S. Cairncross), qui affrontent à l'épée ou à la lance - et non pas au canon qui, historiquement, fit merveille contre les Français durant 
la guerre de Cent Ans - les lâches qui ne savent tuer que de loin ou de l'abri des retranchements ou des murailles. $\mathrm{Du}$ respect des valeurs guerrières pratiquées en un âge lointain, in illo tempore, Talbot, capitaine des troupes anglaises en France, est l'épitomé sublime. C'est un personnage aux vertus univoques. Brave au combat, soucieux d'observer les rites de la chevalerie, fidèle à son roi, il remplit une seule fonction dramatique, proche de la fonction du demi-dieu de la mythologie. Sa force presque surhumaine réduit les Français en hardes aux abois. Il ne prend jamais part aux conflits qui divisent l'aristocratie anglaise. $\mathrm{Au}$ moment des affrontements verbaux entre les clans d'York et de Somerset ou entre Gloucester et Winchester, il guerroie en France ; lorsque Vernon et Basset, Somerset et York poursuivent à Paris les querelles commencées ailleurs, il est déjà «sorti», escorté par ses soldats. C'est autour de lui que la structure de 1 Henri VI-généralement épisodique et vaguement chronologique - se fait architectonique ${ }^{4}$. Ainsi la première scène, qui annonce la capture de ce capitaine, décrit en termes épiques sa magnifique résistance face à un ennemi quatre fois supérieur en nombre :

More than three hours the fight continued ; Where valiant Talbot, above human thought, Enacted wonders with his sword and lance : Hundreds he sent to hell, and none durst stand him ;

Here, there, and everywhere, enrag'd he flew : The French exclaim'd the devil was in arms ;

All the whole army stood agaz'd on him.

(I. i. 120-126)

A l'avant-dernier acte, le même épisode est à nouveau évoqué, et le grandissement mythique est si fort que les compagnons de Talbot se battent maintenant à un contre dix (IV. i, 21)! On remarque le même tour emphatique et répétitif à propos du nom même de Talbot dont un messager revenu des combats annonce qu'il sert de cri 
de guerre à ses soldats et qu'il est perçu comme une fanfare terrifiante par ses ennemis :

His soldiers spying his undaunted spirit, «A Talbot! a Talbot!» cried out amain, And rush'd into the bowels of the battle.

(I. i. 127-129)

Une indication scénique, un acte plus loin, va répéter la chose :

Alarum. Enter an [English] soldier, crying A Talbot! a Talbot! [The French] fly, leaving their clothes behind.

Et si ce n'est pas assez explicite, le soldat va encore commenter :

The cry of «Talbot» serves me for a sword ; For I have loaden me with many spoils, Using no other weapon but his name.

(II. i. 79-81)

Le mythe du guerrier héroïque contamine une bonne partie de la première pièce. Il est vrai que le drame s'ouvre sur l'évocation d'un personnage mythifié :

Henry the Fifth, too famous to live long !

England ne'er lost a king of so much worth.

England ne'er had a king until his time

His brandish'd sword did blind men with his beams :

His arms spread wider than a dragon's wings :

His sparkling eyes, replete with wrathful fire,

More dazzled and drove back his enemies

Than mid-day sun fierce bent against their faces. 
He was a king bless'd of the King of kings.

Unto the French the dreadful judgment-day

So dreadful will not be as was his sight.

(I. i. 6-. . . 30)

Deux douzaines de vers plus loin, le même Henri $\mathrm{V}$ est invoqué comme une divinité :

Henry the Fifth, thy ghost I invocate :

Prosper the realm, keep it from civil broils, Combat with adverse planets in the heavens.

A far more glorious star thy soul will make

Than Julius Caesar or bright -

«Than Julius Caesar or bright. . .» Le vers se brise. L'être quasi-divin aussi glorieux que Jules César et auquel Henri V était bien digne d'être comparé ne sera pas nommé, restera inconnu. Les commentateurs suggèrent que ce nom était illisible dans le MS de 1 Henri $V I^{5}$. Mais ne s'agit-il pas d'une interruption volontaire plutôt que d'une omission? Le nom fut-il jamais écrit ? L'éloge s'arrête abruptement, mais logiquement, à l'arrivée d'un messager venu de France :

Sad tidings bring I to you out of France, Of loss, slaughter, and discomfiture.

Guienne, Champaigne, Rheims, Rouen, Orleans, Paris, Guysors, Poictiers, are all quite lost.

(I. i. 58-61)

Les fières rhétoriques s'éteignent. L'histoire, avec ses échecs, ses défaites, fait taire l'évocation mythique. Les minables muletiers de France ont raison des chevaliers $\mathrm{d}^{\prime}$ Angleterre. Les héros sont introuvables ${ }^{6}$ dans 1 'histoire des Henri VI. Bien plus, cette histoire détruit ses héros potentiels. C'est bien la discorde entre les factions nobiliaires qui précipite la fin du guerrier mythisé par ses exploits, Talbot. Car la haine qui opposait les chefs de clan 
à Londres ou à Paris continue de les diviser, par officier de liaison interposé, près de Bordeaux où Talbot s'apprête à livrer son dernier combat. Des renforts sont réclamés à York puis à Somerset; mais l'un et l'autre se récrient et refusent d'intervenir, car l'occasion pour chacun est trop belle d'accuser le rival détesté d'être responsable de la situation désespérée faite à Talbot. Talbot va se battre sans savoir que ses compatriotes n'ont pas daigné se porter à son secours. L'«idéologie» arriviste d'un Somerset, soucieux de sa seule stratégie personnelle, triomphe des idéaux altiers de Talbot, en qui on a pu voir l'ultime monument d'une société décrépite ${ }^{7}$. Shakespeare modifie alors l'armature du personnage. Il transforme le surhomme presque invincible en un être pathétique abandonné par les Anglais et accablé par un ennemi innombrable. C'est la fin du mythe héroïque et l'amorce d'un autre my the.

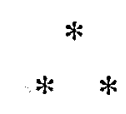

Le pathétique s'accroît avec la venue sur le champ de bataille du fils de Talbot, que Shakespeare, contre 1'histoire, fait apparaître comme un tout jeune homme. Le dramaturge réunit les deux personnages en qui il va réincarner les héros d'un mythe antique, le conte fabuleux d'Ovide sur le vol d'Icare et de Dédale. Avant l'engagement final, Talbot exhorte en effet son fils :

Then follow thou thy desperate sire of Crete, Thou Icarus ; thy life to me is sweet :

If thou wilt fight, fight by thy father's side, And, commendable prov'd, let's die in pride.

(IV. vi. 54-57)

Et lorsqu'on lui apportera la dépouille de John, Talbot évoquera un couple d'êtres ailés fendant le ciel d'azur, après avoir ainsi décrit la mort de son fils :

And in that sea of blood my boy did drench 
His over-mounting spirit ; and there died My Icarus, my blossom, in his pride.

(IV. vii. 13-15)

Talbot-Dédale aura conseillé la fuite à son fils ; un tel vol (flight) n'aura pas eu lieu. $\mathrm{Si}$, dans le récit ovidien, Icare tente d'imiter son père et de copier les gestes qui permettent le vol, le véritable exemple paternel est, dans Shakespeare, infiniment mieux suivi par John que ne l'était par Icare la recommandation de ne pas quitter Dédale des yeux. C'est parce que le jeune Talbot refuse de fuir qu'il imite son père jusqu'au bout. Il y a dans les dialogues entre père et fils, un ton hautement stylisé qui sépare les Talbot du monde historique ambiant. Le choc des notions abstraites, les répétitions qu'accentuent les stychomithies et les rimes, ce duel trop spirituel pour être vraiment touchant et au cours duquel les deux Talbot s'incitent mutuellement mais en vain à la fuite, créent artificiellement un cercle clos à l'intérieur duquel se joue leur tragédie et où tente de renaitre le my the d'Icare.

L'histoire bafouera le mythe et l'écrasera. Les vainqueurs français voudront s'acharner sur les cadavres des Talbot et ne consentiront à rendre les corps que, diront-ils, parce qu'ils commencent à empuantir l'air.

En présentant cette adaptation du mythe d'Icare, Shakespeare a desarticule l'histoire fabuleuse pour la reconstituer dans un cadre évidemment très différent de celui de l'Art d'aimer ou des Métamorphoses. Le dramaturge a hésité devant la tentation facile du vol-fuite et y a renoncé au profit, paradoxalement, du contraire de la fuite : l'engagement dans l'action guerrière qui confère à John Talbot - comme à l'Icare de l'Art d'aimer ou à l'Icare de nombreux emblèmes et poèmes renaissants - l'immortalité de la gloire acquise par un acte d'excessive fierté (pride).

Si l'on est tenté, devant les scènes de la fin des Talbot, de penser que Shakespeare a composé une variation consciente sur le mythe ovidien, force est de juger la deuxième référence à la légende d'Icare et de Dédale, faite dans 3 Henri VI, comme un jeu. 
A la fin de ce drame, Richard de Gloucester pénètre dans la prison de la Tour de Londres pour y tuer l'ex-roi Henri. En apercevant celui qui a déjà tué son fils, le princehéritier des Lancastres, Henri se compare à Dédale, au «malheureux père» qui a maintenant sous les yeux «le fatal objet» par lequel fut tué son fils, "pauvre petit oiseau» (V, vi, 15-17). Richard reconnaît l'allusion et, par dérision, il réfère sur le mode parodique l'action commise (le meurtre du prince) à une sorte de modèle archétypal :

Why, what a peevish fool was that of Crete, That taught his son the office of a fowl!

And yet, for all his wings, the fool was drown'd.

(V. 6. 18-20)

Comme pour conjurer la moquerie des paroles de son ennemi, Henri semble alors se précipiter sur l'allusion mythologique pour la préciser et lui redonner sa dignité :

I, Daedalus ; my poor boy, Icarus ;

Thy father, Minos, that denied our course ;

The sun that sear'd the wings of my sweet boy,

Thy brother Edward; and thyself, the sea

Whose envious gulf did swallow up his life.

(V. 6. 21-25)

Esthétiquement, la référence mythique ne gagne pas grand chose à la différenciation établie par Henri VI entre Edouard (le soleil), Richard (la mer), etc . . . Simplement, la correspondance entre des situations de la pièce et les divers éléments du mythe ovidien (la prison où Minos confinait Dédale et son fils ; le soleil, qui fit fondre la cire des ailes d'Icare ; la mer, où l'enfant se noya) est explicitement énoncée. Or la pièce dans son ensemble prépare mal au réseau d'équations dressées par le roi puisque, par exemple, il arrive au prince, étrange Icare, de conseiller ou même de morigéner son père. Seulement, la référence à l'exemplum mythique permet au personnage de Henri VI d'assumer une attitude qui, il le sait, sera 
la dernière de sa vie et de concentrer, en un bilan hâtif et inexact, les valeurs où il croit exceller ; pitié, amour et pathétique s'y retrouvent.

Ce n'est pas sans mal que Shakespeare a inséré dans les Henri VI ces allusions au mythe d'Icare. Aucun mythe n'est facilement transformable par un créateur littéraire quel qu'il soit ${ }^{8}$. D'une part, le mythe impose, par son rayonnement et son prestige, un respect qui ne saurait s'accomoder d'un bouleversement de ses propres éléments constitutifs. D'autre part, la cohérence d'un drame dépend de l'évolution réussie des personnages, de la progression de l'intrigue, de l'agencement des images et des thèmes - en somme de facteurs nombreux parmi lesquels les références mythiques ne doivent pas apparaître comme de simples adjonctions. C'est peut-être pourquoi la thétorique du my the shakespearien dans Henri VI est si lourde et si particulière. C'est pourquoi aussi il serait possible d'esquisser une topologie du mythe face à l'histoire. De même qu'il existe des espaces différents - espace sacré, espace profane . .. - l'espace mythique existe, lui, face à l'aire historique. Le mythe dans Henri VI éclot, on l'a vu, dans un lieu exceptionnel : à l'écart du combat, en prison ${ }^{9}$...., et les références mythiques s'appliquent à des personnages morts ou qui vont mourir. Outre les passages que j'ai cités, le mythe d'Icare affleure lorsque Clifford, blessé à mort, pressent la chute de sa monarchie (3 Henri VI. II. vi. 5-6); l'histoire mythique d'Etéocle et Polynice n'est pas sans être suggérée par cette rencontre, près du champ de bataille de Towton, entre un père qui a tué son fils et un fils qui a tué son père ( 3 Henri VI. II. v). Dans ces endroits écartés de la fureur des combats et des désordres de l'histoire, hors de l'espace et du temps empiriques, le mythe shakespearien a sa place. Dans le lieu mythique se retrouvent les perdants et les vaincus des Henri VI. La pitié s'y épanche, alors que, dans l'autre espace, seuls comptent ceux qui, sans pitié, usent de la force, du calcul, de la ruse, pour triompher. En ce sens encore, my the et histoire s'opposent dans Henri VI puisque le premier offre un refuge contre la seconde. 


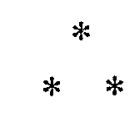

Il arrive pourtant, dans 2 Henri $V I$, que le mythe veuille quitter les lieux marginaux où l'histoire le confine.

Il me semble que l'épisode de la révolte populaire menée par Jack Cade, qui occupe la plus grande partie du plus long des actes de la pièce, est un essai shakespearien d'adaptation du mythe à un contexte réaliste. Lorsque, entrant à Londres en vainqueur, le meneur du Kent frappe de son bâton la borne de la Cité et annonce que désormais le vin coulera à flot des fontaines publiques, il rappelle Moïse frappant le rocher du désert pour en faire jaillir l'eau ${ }^{10}$. La violence du geste proclame l'ouverture d'un espace autre, mythique : l'aire historique, ordinairement peuplée d'une aristocratie en déchéance, doit céder, doit s'ouvrir devant un espace où tout sera possible. Je ne pense pas proposer là un statut trop digne à l'émeute de Cade. Il est vrai que Cade triomphant ressemble à un roi de Carnaval et que ses fantaisies de mégalomane, ses obsessions sanglantes, son décret instaurant à son profit le droit de cuissage, son idée d'une baisse forcée du prix du pain (à moins qu'il ne s'agisse d'un projet d'élévation des mesures de bière) prêtent peut-être à sourire, ou ont peutêtre des résonances sinistres, et sont, en tout cas, dépeints sans le moindre souci d'idéalisation. Mais il serait inexact de ne voir en Cade que l'homme de paille grotesque du duc d'York, qu'un pion manipulé par le prétendant dans son avance vers le pouvoir. Il n'y a en effet que d'infimes rapports entre les dimensions prises par l'émeute et les limites à l'intérieur desquelles le rival du roi lancastrien aurait voulu contenir la fureur populaire. Il n'y a pas, dans la bouche du chef des émeutiers, d'allusions à la connivence qui existe entre lui et Richard d'York. York lui-même ne tire pas, ne tente pas de tirer, avantage des troubles populaires, et ne fait mention du «monstrueux rebelle» qu'une seule fois - et encore après la «déconfiture» de ce dernier. L'alliance du gueux et du duc ne peut donc 
être perçue que comme l'origine lointaine du déferlement. Les indications de la légitimité du mouvement sont, par contre, nombreuses. Gloucester, le bon duc de Gloucester, avait été le protecteur du peuple comme celui du royaume. Il a été assassiné. L'émeute est la manifestation tonitruante d'une contre-justice qui, par la menace de sa force, sait contraindre le roi à sévir contre le principal instigateur du meurtre. La brutalité des hommes de Cade révèle un grossier mais vital désir d' "assommer le crime» et d'《égorger l'iniquité» («Sin struck down like an ox and Iniquity's throat cut like a calf», IV. ii. 26-27, ed. Andrew S. Cairncross). Lorsque les voies de la justice officielle ressemblent, aux dires de celui-même qui est censé l'incarner suprêmement, le roi, au «chemin de l'abattoir sanglant» (III. i. 212), les formes de la lutte contre ce qui n'est qu'une falsification peuvent bien différer de la protestation modérée. Mener à bien une oeuvre de contre-justice, telle est la raison première, immédiate, pour laquelle les Cadiens s'en prennent aux écoles de droit, qu'ils incendient, aux archives du royaume, qu'ils brûlent, au clerc de Chartham, qu'ils exécutent au terme d'un procès parodique - le malheureux est puni pour ce qu'il symbolise, la science juridique, et il meurt pendu, plume et écritoire au cou.

Or le mythe est au coeur de cette frénésie de destruction qui est une négation exacerbée de l'histoire et qui exprime le besoin, sinon de recreer un univers où règne Saturne, un Age d'Or sans lois ni contraintes, du moins d'instaurer un royaume de licence et de richesse - ne serait - ce que celui d'un autre âge d'or où la gloire des armes anglaises permettait aux enfants de jouer à la marelle avec des écus français. Cade fait voeu de tout réformer, se prononce pour un nivellement intégral après le renversement des rois et des princes de la terre. Ses partisans élaborent une théorie de la révolution en raccourci. Cade propose de cette théorie une application - toujours dramatisée sur un mode parodique qui la rend savoureuse-et met en cause les formes qui règlementent les usages. Puisque l'acier symbolise le guerrier noble, il revêt l'armure de Sir Humphrey Stafford qu'il a tué au combat ; puisque l'adou- 
bement confère un titre, il s'arme lui-même chevalier. Puis il annonce l'avènement d'une forme de communisme ; il parle d'abolir l'argent ; il promet l'instauration de la propriété collective ; il décrète une norme vestimentaire qui favorisera l'égalité.

Le temps de la révolte est un temps mythique. Pour, vraisemblablement, accentuer le caractère anhistorique du mouvement, Shakespeare a télescopé les événements et les personnalités de deux soulèvements authentiques, celui de Wat Tyler, en 1381, et celui de Jack Cade, en 1450, créant ainsi une impression de flou temporel. En outre, les Cadiens vivent un moment qu'ils arrachent à l'histoire. Le réalisme, pour eux, serait le retour à l'ordre des choses préexistant, la soumission à un droit honni. C'est d'ailleurs au réalisme que les incitent les premiers envoyés du roi :

Rebellious hinds, the filth and scum of Kent, Mark'd for the gallows, lay your weapons down ; Home to your cottages, forsake this groom. The King is merciful, if you revolt.

(IV. 2. 116-118)

Mais le rêve qui anime les compagnons de Cade est plus grisant que toutes les menaces et que tous les plats appels au calme et au bon sens. Dans le refus d'accepter le retour à une réalité bornée, il y a une sorte de folie mythique au sein de laquelle les hommes jouent avec les idées et avec les mots. Témoin la proclamation «mos̈aïque» de Cade, où culmine le sentiment du magique.

Cade lui-même est un personnage vague et mal défini. Les apartés sarcastiques de certains de ses partisans le présentent comme le fils d'un vagabond ou comme un mendiant ; il aurait subi le fouet; il aurait eu une main brûlée pour vol de bétail. Serait-il drapier de son état, comme l'annonce Bevis, comme le redit Sir Humphrey Stafford, ou soldat des guerres d'Irlande, ainsi qu'avec beaucoup de pittoresque l'a décrit Richard d'York ? Ne serait-il pas l'un des enfants d'Edmond Mortimer, lui qui, encore aux dires d'York, ressemble tant à ce John Mortimer qui 
a disparu ? Prophète inspiré par le Ciel, fils du bas peuple ou prétendant associé aux Mortimer et aux Plantagenêts, comme bien des figures mythiques, il n'a pas de contexte culturel fixe.

La fin de la fureur populaire confirme son caractère mythique en même temps qu'elle fournit un nouveau témoignage du choc entre l'histoire et le mythe. L'apparition de Clifford à la tête d'une nouvelle armée royale administre la preuve de la persistance d'une réalité que les émeutiers voulaient détruire ou dont ils voulaient faire abstraction. Grâce au capitaine dépêché par Henri VI à la rencontre des rebelles, l'histoire va récupérer ses égarés. Remarquons l'habile éloquence de Clifford qui, dans sa harangue, non seulement passe sous silence les conditions politiques catastrophiques dans lesquelles se trouve plongé le royaume et qui ont poussé les hommes du Kent à la révolte, mais surtout évoque un mythe, celui de Henri V, comme s'il s'offrait d'actualiser l'époque de la grandeur anglaise. Le choix qu'il donne aux Cadiens n'est plus entre la soumission à Henri VI et l'aventure avec Cade, mais entre l'aventure avec Cade et le bonheur mythisé de Henri $\mathrm{V}:$

Is Cade the son of Henry the Fifth, That thus you do exclaim you'll go with him? Will he conduct you through the heart of France, And make the meanest of you earls and dukes?

To France, to France! and get what you have lost; Spare England, for it is your native coast.

Henry hath money, you are strong and manly ;

God on our side, doubt not of victory.

(IV. viii. $34-\ldots$ 52)

Bien sûr, Clifford déforme la réalité : il est si loin, le temps des conquêtes. Surtout, il annexe à la cause de la soumission au pouvoir existant des thèmes qui se trouvent au coeur de l'idéologie de l'émeute : désir de promotion et de reconnaissance sociale, nostalgie des gloires, espoir de richesses. . 
Avec lui, l'histoire se sert du mythe. Bientôt le choix des révoltés est fait entre le parti de ceux qui les appellent «concitoyens» et «combattants》 et de celui qui, au comble de la déception, les traite de vils manants.

L'émeute se disperse, le retour à l'histoire est consommé. Le mythe n'aura encore été qu'une parenthèse ; elle se referme. Le mythe se désagrège. Jack Cade n'est plus maintenant un terrifiant meneur mais un hors-latoi traqué et affamé. L'Odyssée chantant le retour à Ithaque ne décrit pas Ulysse comme un homme vieilli par vingt ans de guerre et d'errance, mais toujours comme un héros d'épopée. Au contraire, dans 2 Henri VI, Cade, avant de succomber sous les coups d'un benoît hobereau, broute l'herbe des jardins pour subsister, et son corps, que plusieurs jours de jeûne ont rendu squelettique, inspire presque de la pitié à son vainqueur.

Le mythe, à nouveau, s'est vainement opposé à l'histoire. L'histoire a dévoré le my the.

Pierre SAHEL 
NOTES

1. Les distinctions entre «mythe»et "histoire» présentées dans mon introduction sont en plein accord avec les développements de Moses I:Finley, My the, mém oire, histoire. Les usages du passé (tr.), Paris, Flammarion, 1981.

2. Voir Carol Macginnis Kay, "Traps, Slaughter, and Chaos: : A study of Shakespeare's Henry VI Plays», Studies in the Literary Imagination, V. i. 1972, 1-26.

3. Voir David Riggs, Shakespeare's Heroical Histories : "Henry VI» and its Literary Tradition, Cambridge, Mass., Harvard UP, 1971.

4. Cette réflexion sur la structure de la pièce n'est qu'une incise. Sur les complexités structurales de 1 Henri VI, on lira Kristian Smidt, Unconformities in Shakespeare's History Plays, London, Macmillan, 1982, 18-52.

5. Voir Andrew S. Cairncross, introd. The First Part of King Henry VI, The Arden Shakespeare, London, Methuen, xxiii.

6. Sur le héros introuvable, lire la réflexion de $N$. Rigaud, introd. Le mythe du héros, Aix, Université de Provence, $1982,1$.

7. Michael Manheim, The Weak King Dilemma in the Sha. kespearean History Play, New Y ork, Sy racuse UP, 1973, 97.

8. Voir Raymond Trousson, «Servitude du créateur en face du mythe", Cahiers de l'Association internationale des études fran. faises, 20, M ai $1968,85-98$.

9. Sur la prison, lieu paradoxalement priviligié, on peut lire Pierre Sahel, "Les prisons politiques chez Marlowe et Shakespeare», Société française Shakespeare, Actes du Congrès 1980,1981, en particulier 108 .

10. François Laroque, «Shakespeare et la fête populaire : Le carnaval sanglant de Jack Cade», Bulletin de l'Association d'étude sur l'Hum anisme, la Réforme et la Renaissance, VI, 11, 129. 